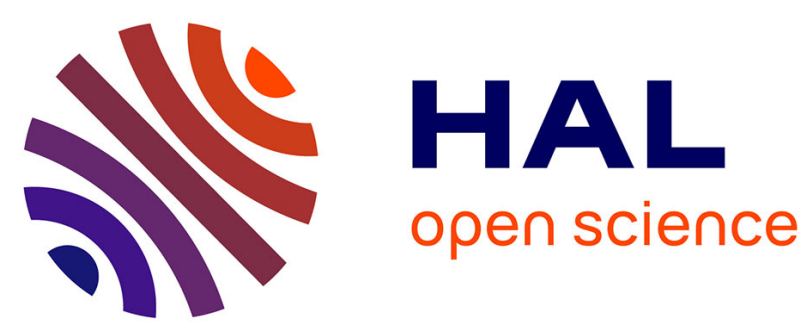

\title{
Le site de référence du Partenariat européen d'innovation pour un vieillissement actif et en bonne santé MACVIA-LR (contre les maladies chroniques pour un vieillissement en bonne santé en Languedoc-Roussillon)
}

Jean Bousquet, Rodolphe Bourret, Thierry Camuzat, Philippe Augé, Philippe Domy, Jacques Bringer, Nicolas Nicolas, Olivier Jonquet, Jean-Emmanuel de La Coussaye, Michel Noguès, et al.

\section{To cite this version:}

Jean Bousquet, Rodolphe Bourret, Thierry Camuzat, Philippe Augé, Philippe Domy, et al.. Le site de référence du Partenariat européen d'innovation pour un vieillissement actif et en bonne santé MACVIA-LR (contre les maladies chroniques pour un vieillissement en bonne santé en LanguedocRoussillon). La Presse Médicale, 2015, 44 (1), pp.S6-S22. 10.1016/j.lpm.2015.07.013 . hal-01756022

\section{HAL Id: hal-01756022 \\ https://hal.umontpellier.fr/hal-01756022}

Submitted on 6 May 2020

HAL is a multi-disciplinary open access archive for the deposit and dissemination of scientific research documents, whether they are published or not. The documents may come from teaching and research institutions in France or abroad, or from public or private research centers.
L'archive ouverte pluridisciplinaire HAL, est destinée au dépôt et à la diffusion de documents scientifiques de niveau recherche, publiés ou non, émanant des établissements d'enseignement et de recherche français ou étrangers, des laboratoires publics ou privés. 


\section{Le site de référence du Partenariat européen d'innovation pour un vieillissement actif et en bonne santé MACVIA-LR (contre les maladies chroniques pour un vieillissement en bonne santé en Languedoc-Roussillon)}

Groupe d'étude MACVIA-LR, Jean Bousquet ${ }^{1,2}$, Rodolphe Bourret $^{1}$, Thierry Camuzat ${ }^{3}$, Philippe Augé ${ }^{4}$, Philippe Domy ${ }^{1}$, Jacques Bringer ${ }^{5}$, Nicolas Best ${ }^{6,113}$, Olivier Jonquet ${ }^{7}$, Jean-Emmanuel de la Coussaye ${ }^{8}$, Michel Noguès ${ }^{2,9}$, Jean-Marie Robine ${ }^{10,11,12}$, Antoine Avignon ${ }^{13}$, Hubert Blain ${ }^{14,15}$, Bernard Combe ${ }^{16}$, Gérard Dray ${ }^{17}$, Vincent Dufour ${ }^{18}$, Mireille Fouletier ${ }^{17}$, Nicolas Giraudeau ${ }^{19}$, Didier Hève ${ }^{20}$, Claude Jeandel ${ }^{14}$, Isabelle Laffont ${ }^{15,21}$, Dominique Larrey ${ }^{22}$, Daniel Laune ${ }^{23}$, Cyril Laurent ${ }^{24}$, Pierre Mares ${ }^{25}$, Chantal Marion ${ }^{26}$, Eric Pastor ${ }^{27,28}$, Jacques-Yvon Pélissier ${ }^{15,29}$, Françoise Radier-Pontal ${ }^{30}$, Jacques Reynes ${ }^{31,32}$, Emilie Royère ${ }^{33}$, Marc Ychou ${ }^{34}$, Anna Bedbrook ${ }^{1,2}$, Sophie Granier ${ }^{8}$, Frédéric Abecassis ${ }^{35}$, Sylvie Albert ${ }^{36}$, Paule Adnet $^{14}$, Bernard Alomène ${ }^{14}$, Michel Amouyal ${ }^{37}$, Sylvie Arnavieilhe ${ }^{23}$, Trias Asteriou ${ }^{26}$, Vincent Attalin ${ }^{38}$, Pierre Aubas ${ }^{39}$, Christine Azevedo ${ }^{40}$, Mélanie Badin ${ }^{37}$, Karima Bakhti ${ }^{15,21}$, Gregory Baptista ${ }^{14}$, Benoit Bardy ${ }^{15}$, Marie-Pierre Battesti ${ }^{20}$, Olivier Bénézet ${ }^{41}$, Pierre-Louis Bernard ${ }^{15}$, Claudine Berr ${ }^{42}$, Jacquie Berthe ${ }^{33}$, Xavier Bobia ${ }^{8}$, Joël Bockaert ${ }^{43}$, Catherine Boegner ${ }^{13}$, Sylvie Boichot ${ }^{44}$, Huei-Yune Bonnin ${ }^{15,29}$, Philippe Boulet ${ }^{26}$, Stéphane Bouly ${ }^{8}$, Chokri Boubakri ${ }^{14}$, Arnaud Bourdin ${ }^{45}$, Jean-Luc Bourrain ${ }^{45}$, Gérard Bourrel ${ }^{37}$, Vincent Bouix ${ }^{46}$, Cyril Breuker ${ }^{1}$, Valérie Bruguière ${ }^{9}$, Jacques Burille ${ }^{47}$, Stéphane Cade ${ }^{48}$, Davide Caimmi ${ }^{45}$, Marie-Virginie Calmels ${ }^{49}$, William Camu ${ }^{50}$, Gérard Canovas ${ }^{51}$, Véronique Carre ${ }^{21}$, Giacomo Cavalli ${ }^{52}$, Guillaume Cayla ${ }^{53}$, Raphaël Chiron ${ }^{45}$, Pierre-Géraud Claret ${ }^{8}$, Pauline Coignard ${ }^{54}$, Flavia-Oana Coroian ${ }^{15,21}$, David Costa ${ }^{37}$, Pierre Costa ${ }^{55}$, Jérome Cottalorda ${ }^{56}$, Bertrand Coulet ${ }^{57}$, Anne-Laure Coupet ${ }^{9}$, Marie-Christine Courrouy-Michel ${ }^{58}$, Philippe Courtet $^{58}$, Jean-Paul Cristol ${ }^{59}$, Valérie Cros ${ }^{21}$, Frédéric Cuisinier ${ }^{60}$, Claire Daien ${ }^{16}$, Marianna Danko ${ }^{58}$, Philippe Dauenhauer ${ }^{61}$, Michel Dauzat ${ }^{62}$, Miren David ${ }^{37}$, Jean-Marc Davy ${ }^{48}$, Didier Delignières ${ }^{15}$, Pascal Demoly ${ }^{45}$, Matthieu Desplan ${ }^{63}$, Henriette Dhivert-Donnadieu ${ }^{45}$, Pierre Dujol ${ }^{49}$, Arnaud Dúpeyron ${ }^{15,29}$, Gérard Dupeyron ${ }^{64}$, Oude Engberink ${ }^{15,65}$, Michel Enjalbert ${ }^{54,66}$, Charles Fattal ${ }^{15,54,67}$, Jérôme Fernandes ${ }^{68}$, Pierre Fesler ${ }^{69}$, Philippe Fraisse ${ }^{40}$, Jérôme Froger ${ }^{15,8}$, Philippe Gabrion ${ }^{70}$, Emilie Galano ${ }^{29}$,

Marion Gellerat-Rogier ${ }^{71}$, Anthony Gellis ${ }^{67}$, Arnaud-Yves Goucham ${ }^{18}$, Fares Gouzi ${ }^{72}$, Florence Gressard ${ }^{27}$, Jean-Christophe Gris ${ }^{73}$, Bernard Guillot ${ }^{74}$, David Guiraud ${ }^{40}$, Valérie Handweiler ${ }^{1}$, Olivier Hantkié ${ }^{75}$, Maurice Hayot ${ }^{72}$, Christian Hérisson ${ }^{15,21}$, Cherif Heroum ${ }^{1}$, Didier Hoa ${ }^{22}$, Sylvain Jacquemin ${ }^{76}$, Samir Jaber ${ }^{77}$, Dominique Jakovenko ${ }^{78}$, Christophe Jorgensen ${ }^{16,79}$, Laurent Journot ${ }^{43}$, Michel Kaczorek ${ }^{32}$, Pascal Kouyoumdjian ${ }^{80}$, Pierre Labauge ${ }^{50}$, Liliane Landreau ${ }^{7}$, Martine Lapierre ${ }^{29}$, Catherine Leblond ${ }^{66}$, Marie-Suzanne Léglise ${ }^{14}$, Jean-Marc Lemaitre ${ }^{79}$, Vincent Le Moing ${ }^{31,32}$, Alain Le Quellec ${ }^{69}$, Françoise Leclercq ${ }^{48}$, Sylvain Lehmann ${ }^{81}$, Béatrice Lognos ${ }^{37,82}$, Jean-Marc Lussert ${ }^{83}$, Alain Makinson ${ }^{31}$, Kevin Mandrick ${ }^{15}$, Vincent Marmelat ${ }^{15}$, Pierre Martin-Gousset ${ }^{27}$, Amélie Matheron ${ }^{49}$, Gwen Mathieu ${ }^{37}$, Marc Meissonnier ${ }^{84}$, Grégoire Mercier ${ }^{85}$, Patrick Messner ${ }^{53}$, Cyril Meunier ${ }^{27,86}$, Michél Mondain ${ }^{87}$, Raul Punta-Morales ${ }^{50}$, jacques Morel ${ }^{16}$, David Morquin ${ }^{31,32}$, Denis Mottet ${ }^{15}$, Philippe Nérin ${ }^{88}$, Pierre Nicolas ${ }^{75}$, Gregory Ninot ${ }^{65}$, Fabrice Nouvel ${ }^{29,89,90}$, Jean-Paul Ortiz ${ }^{91}$, Delphine Paccard ${ }^{9}$, Guillaume Pandraud ${ }^{30}$, Marie-Pierre Pasdelou ${ }^{49}$, Jean-Luc Pasquié ${ }^{48}$, Karine Patte ${ }^{92}$, Stéphane Perrey ${ }^{15}$, 
Yves-Marie Pers ${ }^{16}$, Marie-Christine Picot ${ }^{39}$, Jean-Philippe Pin ${ }^{43}$, Nathalie Pinto ${ }^{9}$, Emilie Porte ${ }^{29}$ Fabienne Portejoie ${ }^{2}$, Jean-Louis Pujol ${ }^{45}$, Xavier Quantin ${ }^{45}$, Isabelle Quéré ${ }^{69}$, Nathalie Raffort ${ }^{47}{ }^{\prime}$ Sofiane Ramdani ${ }^{14}$, Jean Ribstein ${ }^{69}$, Isabelle Rédini-Martinez ${ }^{20}$, Sylvain Richard ${ }^{93}$, Karen Ritchie ${ }^{94}$, Jean-Pierre Riso ${ }^{95}$, François Rivier ${ }^{96}$, Christine Rolland ${ }^{97}$, François Roubille ${ }^{48}$, Denis Sablot ${ }^{98}$, Jean-Luc Savy ${ }^{49}$, Laurent Schifano ${ }^{92}$, Pierre Senesse ${ }^{34}$, Roland Sicard ${ }^{99}$, Baya Soua ${ }^{100}$, Yannick Stephan ${ }^{65}$, Denise Strubel ${ }^{101}$, Ariane Sultan ${ }^{12}$, Roxana Taddei-Ologeanu ${ }^{102}$, Guillaume Tallon ${ }^{14}$, Michel Tanfin ${ }^{95}$, Hervé Tassery ${ }^{103}$, Isabel Tavares ${ }^{21}$, Kjerstin Torre ${ }^{14}$, Jacques Touchon ${ }^{9,31}$, Vincent Tribout ${ }^{31}$, Alain Uziel ${ }^{87}$, Philippe Van de Perre ${ }^{104}$, Xavier Vasquez ${ }^{105}$, Jean-Michel Verdier ${ }^{106,107,108}$, Céline Vergne-Richard ${ }^{20}$, Grégoire Vergotte ${ }^{15}$, Laurence Vian ${ }^{109}$, Christine Viarouge-Reunier ${ }^{1}$, François Vialla ${ }^{110}$, Frédéric Viart ${ }^{111}$, Max Villain ${ }^{112}$, Maxime Villiet ${ }^{1}$, Emilie Viollet ${ }^{15,29}$, Anne Wojtusciszyn ${ }^{13}$, Martine Aoustin ${ }^{20}$, Christian Bourquin ${ }^{18,114}$, Jacques Mercier ${ }^{4,72,93}$

1. CHRU de Montpellier, 34295 Montpellier cedex 5, France

2. MACVIA-LR, contre les maladies chroniques pour un vieillissement actif en Languedoc-Roussillon, European Innovation Partnership on Active and Healthy Ageing Reference Site, 34000 Montpellier, France

3. Région Languedoc-Roussillon, 34000 Montpellier, France

4. Université de Montpellier, 34000 Montpellier, France

5. Faculté de médecine, 34000 Montpellier-Nîmes, France

6. CHRU de Montpellier, département de réanimation, 34295 Montpellier, France

7. CHRU de Nîmes, département de réanimation, 30000 Nîmes, France

8. CHRU de Nîmes, 30000 Nîmes, France

9. Caisse assurance Retraite et santé au travail Languedoc-Roussillon (CARSAT-LR), 34295 Montpellier, France

10. Inserm U 988, 75000 Paris, France

11. Inserm U 710, 34000 Montpellier, France

12. École pratique des Hautes Études (EPHE), 75000 Paris, France

13. CHRU de Montpellier, département d'endocrinologie, 34000 Montpellier, France

14. CHRU de Montpellier, départment de gériatrie, 34295 Montpellier, France

15. Université de Montpellier, EA 2991 Movement To Health, Euromov, 34000 Montpellier, France

16. CHRU de Montpellier, département de rhumatologie, 34295 Montpellier, France

17. École des mines, 30100 Alès, France

18. Région Languedoc-Roussillon, 34000 Montpellier, France

19. Université de Montpellier, école dentaire, 34000 Montpellier, France

20. Agence régionale de santé, 34000 Montpellier, France

21. CHRU de Montpellier, département de médecine physique et de réadaptation, 34295 Montpellier, France

22. CHRU de Montpellier, département d'hépatologie, 34295 Montpellier, France

23. Kyomed, 34000 Montpellier, France

24. I2ML, fondation institut méditerraneen des métiers de la longévité, 30000 Nîmes, France

25. CHRU de Nîmes, département de gynécologie-obstétrique, 30000 Nîmes, France

26. Agglomération de Montpellier, 30000 Montpellier, France

27. L'ÉTAPE - pôle autonomie santé, CCAS de Lattes, 34970 Lattes, France

28. Conseil régional de l'ordre des masseurs kinésithérapeutes, maison des professions libérales, 34000 Montpellier, France

29. CHRU de Nîmes, hôpital Carémeau et du Grau du Roi, département de médecine physique et de réadaptation, 30000 Nîmes, France

30. Conseil départemental de l'ordre des pharmaciens, maison des professions libérales, 34000 Montpellier, France

31. CHRU de Montpellier, département d'infectiologie, 34295 Montpellier, France

32. IRD-université de Montpellier, unité mixte internationale 233 " recherches translationnelles sur l'infection VIH et les maladies infectieuses », 34000 Montpellier, France

33. Pôle de compétitivité Eurobiomed PAC-Languedoc-Roussillon, 13000 Marseille, France

34. Institut du Cancer, 34000 Montpellier, France

35. Fédération pharmaceutique méditerranéenne (FEDMED), 34320 Roujan, France

113 Adresse (Directeur Général, CH Annecy).

114 Décédé. 
36. Agropolis international, 34000 Montpellier, France

37. Faculté de médecine de Montpellier, département de médecine générale, 34000 Montpellier, France

38. Aviitam, 34000 Montpellier, France

39. CHRU de Montpellier, département de santé publique, 34295 Montpellier, France

40. Université de Montpellier, et institut national de recherche en informatique et en automatique, LIRMM, laboratoire d'informatique, de robotique et de microélectronique de Montpellier, 34000 Montpellier, France

41. 30000 Nîmes, France

42. Inserm, U1061, 34000 Montpellier, France

43. CNRS, institut de génomique fonctionnelle, 34000 Montpellier, France

44. Transferts LR, 34000 Montpellier, France

45. CHRU de Montpellier, département des maladies respiratoires et addictologie, 34000 Montpellier, France

46. CHG d'Alès, 30100 Alès, France

47. Société publique locale d'exploitation de Balaruc-les-Bains, 34540 Balaruc-lesBains, France

48. CHRU de Montpellier, départment de cardiology, 34000 Montpellier, France

49. Mairie de Juvignac, centre communal d'action sociale, 34990 Juvignac, France

50. CHRU de Montpellier, département de neurologie, 34295 Montpellier, France

51. 34540 Ballaruc-les-Bains, France

52. CNRS, institut de génétique humaine, 34000 Montpellier, France

53. CHRU de Nîmes, département de cardiologie, 30000 Nîmes, France

54. CMRRF de Kerpape, association APPROCHE, 56270 Ploemeur, France

55. CHRU Nîmes, département d'urologie 30000 Nîmes, France

56. CHRU de Montpellier, département de chirurgie orthopédique et plastique infantile, 34295 Montpellier, France

57. CHRU de Montpellier, département de chirurgie orthopédique, 34295 Montpellier, France

58. CHRU de Montpellier, département de psychiatrie, 34000 Montpellier, France

59. CHRU de Montpellier, département de biologie, 34295 Montpellier, France

60. LBN EA 4203, 34000 Montpellier, France

61. Sanofi-Aventis, 34000 Montpellier, France

62. CHRU de Nîmes, département de physiologie, 30000 Nîmes, France

63. Fontalvie, 34000 Montpellier, France

64. CHRU de Nîmes, département d'ophtalmologie, ARAMAV (Association réinsertion aveugles malvoyants), 30000 Nîmes, France

65. Université de Montpellier, EA4556 Epsylon, 34000 Montpellier, France

66. Centre Bouffard Vercelli, 66290 Cerbère, France

67. Centre mutualiste propara, 34000 Montpellier, France

68. Oc santé, 34000 Montpellier, France

69. CHRU de Montpellier, département de médecine interne, 34000 Montpellier, France

70. Languedoc-Roussillon incubation, UM2, 34000 Montpellier, France

71. 34000 Montpellier, France

72. CHRU de Montpellier, département de physiologie, 34000 Montpellier, France

73. CHRU de Nîmes, département de biologie, 30000 Nîmes, France

74. CHRU de Montpellier, département de dermatologie, 34295 Montpellier, France

75. Centre Bourgès, groupe oc santé, 34000 Montpellier, France

76. Horiba, 34000 Montpellier, France

77. CHRU de Montpellier, département d'anesthésiologie, 34295 Montpellier, France

78. Union régionale des professions de soins infirmiers libéraux du LanguedocRoussillon, maison des professions libérales, 34000 Montpellier, France

79. Institut de recherche en biothérapie, 34000 Montpellier, France

80. CHU Carémeau, département de chirurgie orthopédique, 30000 Nîmes, France

81. Institut de médecine régénératrice et de biothérapie (I.M.R.B.), University hospital, Inserm U1040, institut de génétique humaine, CNRS- UPR1142, 34000 Montpellier, France

82. Union régionales de professions de soins médecins libéraux du LanguedocRoussillon, maison des professions libérales, 34000 Montpellier, France

83. Mairie, centre communal d'action sociale (CCAS), 34730 Prades-Le-Lez, France

84. Anduze, 30140 France

85. CHRU de Montpellier, unité médico-économie, département de l'information médicale, 34295 Montpellier, France

86. Agglomération de Montpellier, 34000 Montpellier, France

87. CHRU de Montpellier, département d'ORL, 34000 Montpellier, France

88. SATT AxLR, 34000 Montpellier, France

89. Association francaise des ergothérapeutes francais, 34160 Boisseron, France

90. CHRU de Nîmes, clinique du positionnement et de la mobilité, 30000 Nîmes, France

91. Union nationale de professions de soins médecins libéraux, 75015 Paris, France

92. Institut Marin-Saint-Pierre, 34250 Palavas-les-Flots, France 
93. CNRS, université de Montpellier, Inserm U1046, physiologie \& médecine expérimentale, cœur et muscles, 34000 Montpellier, France

94. Inserm U1061 neuropsychiatrie, Montpellier, Faculty of Medicine, Imperial College, London, Royaume-Uni

95. ADMR Gard, 30000 Nîmes, France

96. CHRU de Montpellier, département de neuropédiatrie, centre de référence Grand-Sud des maladies neuro-musculaires, 34000 Montpellier, France

97. Association asthme et allergie, 92100 Boulogne, France

98. CHG, 66000 Perpignan, France

99. La Valériane, 34000 Montpellier, France

100. Association ADAGES, Les Passerelles d'ô, 34000 Montpellier, France

101. CHRU de Nîmes, département de gériatrie, 30000 Nîmes, France

102. Université Montpellier 2, laboratoire de recherche système d'information Montpellier Management recherche, 34000 Montpellier, France

103. LBN EA 4203, 34000 Montpellier, France

104. CHRU et Inserm U 1058, 34295 Montpellier, France

105. IBM, 34000 Montpellier, France

106. EPHE, section des sciences de la vie et de la terre, 75000 Paris, France

107. UMR S 710, université de Montpellier, 34000 Montpellier, France

108. Institut transdisciplinaire d'études du vieillissement, 34000 Montpellier, France

109. Université de Montpellier, faculté de pharmacie, 34000 Montpellier, France

110. CEERDS UMR 5815, UFR droit et science politique, 34000 Montpellier, France

111. ASA - Advanced Solutions Accelerator, 34830 Clapiers, France

112. CHRU de Montpellier, département d'ophtalmologie, 34000 Montpellier, France

Correspondance :

Jean Bousquet, CHRU de Montpellier, 34295 Montpellier cedex 5, France.

jean.bousquet@orange.fr

Union européenne connaît un vieillissement démographique considérable. Depuis 2012, la population européenne en âge de travailler se réduit, tandis que celle de plus de 60 ans poursuit sa progression. Cette évolution va mettre en péril les futurs équilibres financiers publics dont celui des soins de santé [1].

L'espérance de vie à la naissance ne cesse d'augmenter dans le monde, approchant ou dépassant 85 ans pour les femmes et 80 ans pour les hommes en Europe. Cette évolution est due à l'allongement de la longévité moyenne et non à la baisse de la mortalité prématurée. La qualité de vie des années supplémentaires, vécues après 80 ou 85 ans, préoccupe de plus en plus les pouvoirs publics qui cherchent à retarder l'apparition des maladies chroniques, la sénescence, le déclin fonctionnel ainsi que la fragilité et la perte d'autonomie [2]. Désormais, la prise en compte par l'Union européenne de l'espérance de vie en bonne santé (sans maladie chronique invalidante et sans limitations d'activité) dans le calcul de l'espérance de vie globale permet d'apprécier le poids du vieillissement [3].

Les maladies chroniques jouent un rôle majeur dans le vieillissement [4]. L'augmentation de l'espérance de vie s'accompagne d'un changement des causes de morbidité et de mortalité, plus de $70 \%$ étant désormais imputables aux maladies chroniques [5]. L'augmentation du nombre de personnes atteintes de maladies chroniques génère de nouveaux risques liés aux interactions entre de multiples pathologies (comorbidités), qui s'ajoutent aux réductions fonctionnelles liées à l'âge et aux causes socio-économiques [6].
La Commission européenne (DG-Sanco et DG-CNECT) a lancé un appel de déclarations d'intention pour les candidatures de " site de référence " dans le cadre du Partenariat européen d'innovation pour un vieillissement actif et en bonne santé (European Innovation Partnership on Active and Healthy Ageing - EIP on $A H A$. Ces sites sont définis comme étant des régions, villes ou organisations intégrées (entre les hôpitaux et les soins de santé primaires) qui, par une approche innovante et globale, permettront d'améliorer le vieillissement actif et en bonne santé. Ils doivent intégrer plusieurs actions. Trente-deux sites de référence, dont quatre régions ou départements français, ont été retenus et ont reçu une reconnaissance officielle le $1^{\text {er }}$ juillet 2013. Рarallèlement, la Commission a lancé un appel d'offre concernant les " engagements pour action » (Commitment for Action).

Cet article résume les activités en région Languedoc-Roussillon (LR) qui ont abouti à la création du site de référence $M A C V I A-L R^{\circledR}$ (contre les maladies chroniques pour un vieillissement actif en Languedoc-Roussillon) ainsi qu'à celle de l'institut EURICAP (European Institute for Chronic Diseases, Ageing and Handicap). Ces deux entités sont fondées sur les appels d'offre de la Commission européenne [7]. MACVIA-LR ${ }^{\circledR}$ [8] et EURICAP se proposent de représenter les activités du site co-localisé français de OneMoreLife en interaction avec les autres partenaires français.

\section{Le partenariat européen d'innovation pour le vieillissement actif et en bonne santé}

Le but du Partenariat européen d'innovation pour un vieillissement actif et en bonne santé (EIP on AHA, DG-Sanco et 


\section{ENCADRÉ 1}

\section{Plan d'action de I'EIP on AHA}

A1 Meilleure adhésion aux prescriptions de médicaments Aujourd'hui, seul un quart des sujets âgés ayant une maladie chronique avec comorbidités prennent correctement leur traitement. La Commission propose de développer des approches innovantes dans au moins 30 régions pour améliorer l'adhésion aux traitements

A2 Prévention des chutes Les chutes représentent une morbidité et une mortalité très importantes chez les sujets âgés. Elles sont aussi responsables de perte d'autonomie. Un programme de prévention des chutes et de diagnostic précoce devrait être lancé dans au moins 10 pays d'Europe en 2015 . La télémédecine doit pouvoir aider à détecter et prévenir les chutes. Des programmes de réhabilitations seront mis en place.

A3 Fragilité et malnutrition Un programme pour la prévention du déclin fonctionnel et la fragilité doit être mis en place et proposé à 1000 individus au minimum parmi le personnel de santé, à compter de 2015

B3 Prise en charge intégrée des soins pour les maladies chroniques avec comorbidités

Des programmes pour les sujets âgés seront mis en place dans au moins 20 régions pour 2015. Ces programmes seront focalisés sur l'intégration des soins par les professionnels de santé et sociaux. Ils incluront la télémédecine

C2 Vie autonome

La mise en place et l'intégration de solutions entre divers types de technique d'aide et d'intervenants, pour maintenir les sujets âgés à domicile, doivent inclure un ensemble de mesures comme : les contacts sociaux, les alarmes fonctionnelles et divers services à domicile. Les sujets âgés doivent apprendre à utiliser les applications de télémédecine, faciles à comprendre, pour prévenir la solitude, l'isolement social ou l'admission prématurée dans une maison de retraite

D4 Environnement adapté aux personnes âgées, appartements thérapeutiques et villes amies

Les villes, villages et les zones publiques doivent s'adapter aux personnes âgées et/ou handicapées pour permettre à toute personne de ne pas rester confinée chez elle

DG-CNECT ; https://webgate.ec.europa.eu/eipaha/) est de mobiliser les acteurs et les ressources en Europe pour identifier et éliminer les freins à l'innovation en faveur d'un vieillissement actif et en bonne santé. Par une approche interdisciplinaire et intersectorielle, la création de synergies et d'une masse critique impliquant les secteurs privés et publics à tous les niveaux de la société (local/régional/national/européen), il s'est donné pour mission de dessiner les contours d'une société soucieuse d'assurer un vieillissement actif et en bonne santé de sa population. L'objectif phare est d'augmenter de deux ans les années de vie en bonne santé
TABLEAU I

Actions initiées en LR pour l'EIP on AHA

\begin{tabular}{lcc} 
& $\begin{array}{c}\text { Vieillissement } \\
\text { actif }\end{array}$ & $\begin{array}{c}\text { Mieux vieillir } \\
\text { avec une } \\
\text { maladie }\end{array}$ \\
\hline Prévention des chutes & +++ & +++ \\
\hline Clinique de comorbidités & - & ++ \\
\hline Maladies respiratoires chroniques & +++ & ++ \\
\hline Urgences & +++ & +++ \\
\hline Soins dentaires & ++ & +++ \\
\hline Maladies infectieuses chroniques & +++ & ++ \\
\hline Hépatites & + & +++ \\
\hline Mieux vieillir avec un cancer & + & +++ \\
\hline Mieux vieillir avec une mucoviscidose & - & +++ \\
\hline
\end{tabular}

(Healthy Life Years) des citoyens de l'Union européenne (UE) d'ici 2020 [9]. Le succès d'une telle démarche conduirait à une triple victoire pour l'Europe en :

- permettant aux citoyens de l'UE de mener une vie saine, active et autonome jusqu'à un âge avancé ;

- assurant la pérennisation et l'efficacité des systèmes de soins sociaux et de santé ;

- développant et déployant des solutions innovantes, moteurs de la compétitivité et de la croissance du marché.

L'EIP on AHA a identifié une série de plans d'action, regroupés en trois piliers:

- A : prévention et dépistage ;

- B : soins et traitements ;

- C/D : vieillissement actif et vie autonome) (encadré 1).

La Commission européenne a créé des sites de références de I'EIP on AHA. Trente-deux, dont quatre régions ou départements français, ont été retenus et ont reçu une reconnaissance officielle le $1^{\text {er }}$ juillet 2013. La Commission a également lancé un appel d'offre concernant les « engagements pour action » (Commitment for Action).

\section{MACVIA-LR ${ }^{\circledR}$, site de référence de I'EIP on AHA}

Le 2 mars 2012, I'EIP on AHA a lancé plusieurs appels d'offre pour un plan stratégique. La région Languedoc-Roussillon (LR) a répondu à I' " expression d'intérêt » pour un site de référence candidats (plans d'action A1, A2, A3, B3, C2 et D4) et 10 engagements pour action (A2, B3 et D4). Les projets du LR ont tous été retenus par I'Union européenne (5 en LR et 5 en Europe) (tableau I). 


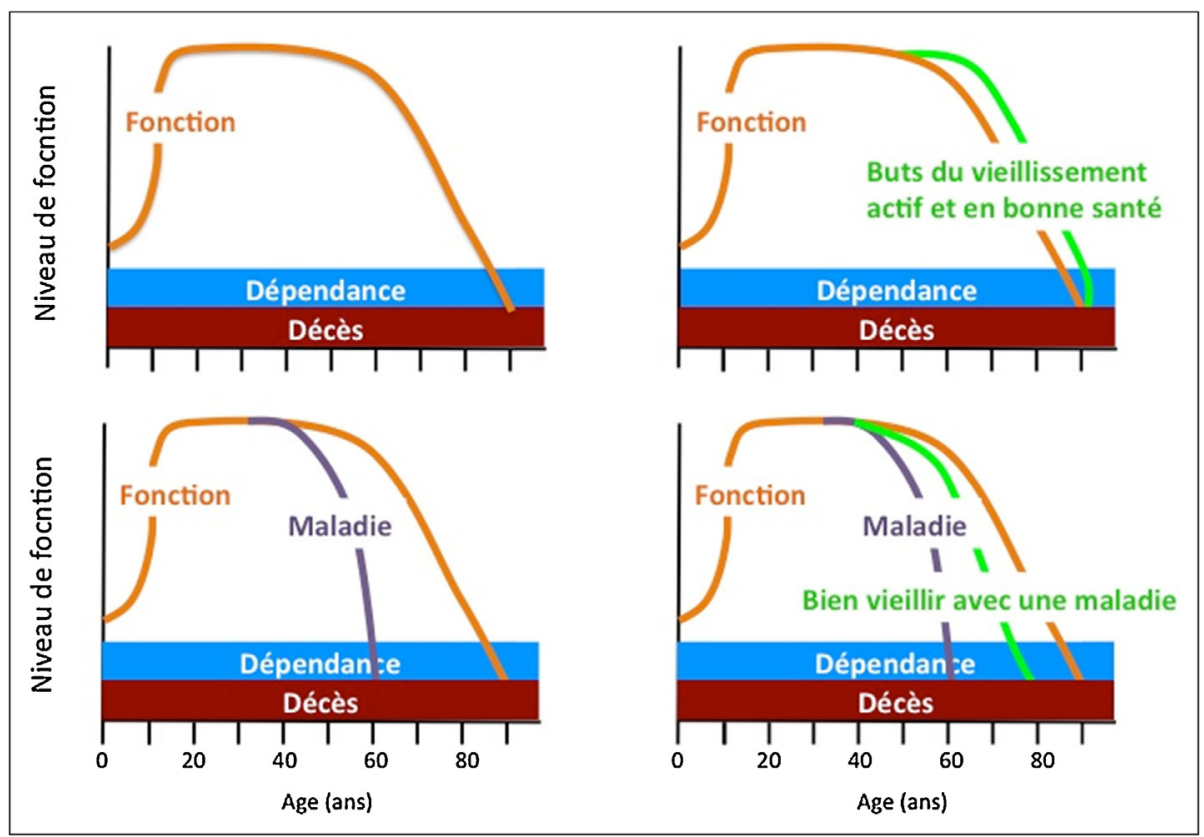

FIGURE 1

Vision du Site de référence MACVIA-LR

La vision du site de référence est double : permettre, d'une part, de vieillir en étant actif et en bonne santé et, d'autre part, de mieux vieillir en étant malade (figure 1).

\section{Plan d'action A1 : adhésion aux prescriptions de médicaments}

Les pharmaciens représentent un atout important du système de soins français. Une interaction avec l'Ordre des pharmaciens, les associations professionnelles et les maîtres de stages a été initiée, en particulier pour intégrer le Dossier Pharmaceutique ${ }^{\circledR}$ au projet et pour une meilleure adhésion au traitement des personnes âgées. Les autres acteurs des soins auprès des personnes âgées (aides-soignantes et autres) sont aussi impliqués pour optimiser les soins à domicile.

\section{Plan d'action A2 : prévention des chutes}

Les chutes représentent une morbidité et une mortalité très importantes chez les sujets âgés [10]. Elles sont aussi responsables de perte d'autonomie. La clinique de la prévention des chutes fonctionne au CHRU de Montpellier et sera déployée dans les CHG de la région [11]. L'information sera faite au niveau des médecins de la région mais aussi des pharmaciens. Un réseau de soins doit être établi pour prévenir les chutes.

\section{Plan d'action A3 : fragilité et malnutrition}

La fragilité est un des axes majeurs de la gériatrie pour prévenir le handicap et la perte d'autonomie [12]. L'Institut régional du vieillissement de la CARSAT-LR (caisse d'assurance retraite et de la santé au travail-LR) est au centre du dispositif de la fragilité car on insiste insuffisamment sur les éléments déterminants socio-économiques de la prise en charge de la fragilité. Le projet Trans-Innov-Longévité assure le déploiement d'un enseignement à distance pour la formation aux nouveaux métiers dans le champ du vieillissement et prévenir la fragilité. Le parcours de santé des personnes âgées en risque de perte d'autonomie (PAERPA) entre aussi dans le cadre du plan d'action A3 et sa mise en place est en cours au CHRU de Montpellier.

\section{Plan d'action B3 : prise en charge intégrée des soins pour les maladies chroniques avec comorbidités}

Les maladies chroniques sont des affections de longue durée. Elles associent les quatre pathologies non transmissibles majeures définies par l'OMS (maladies cardiovasculaires, cancers, maladies respiratoires chroniques, diabète) [13] mais aussi les maladies neuropsychiatriques, musculo-squelettiques et la santé bucco-dentaire [14]. Plusieurs maladies infectieuses sont des affections chroniques (tuberculose, VIH/SIDA). Enfin, les maladies génétiques sont devenues des maladies chroniques. Un des meilleurs exemples en est la mucoviscidose (figure 2).

Le parcours de soins pour les maladies chroniques et le vieillissement actif et en bonne santé nécessite des propositions innovantes intégrant les acteurs publics et privés autour des soins en santé primaire et l'implication des patients. Il doit être applicable à l'ensemble de la région, en tenant compte des inégalités sociales et géographiques ainsi que de la stratégie régionale de l'accès aux soins pour tous. Les propositions : 


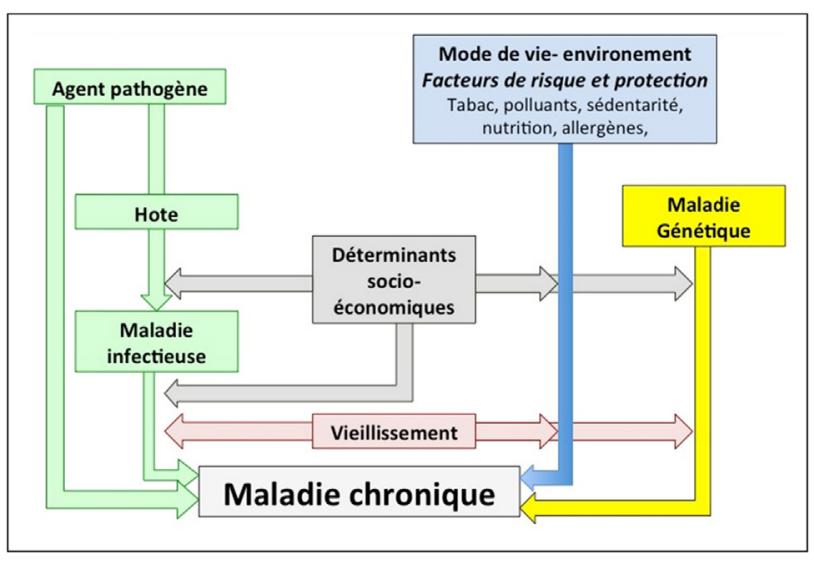

FIGURE 2

Les maladies chroniques (adapté de [15])

- offre de soins intégrée et complémentaire pour les maladies chroniques avec comorbidités (CHRU de Montpellier). Un projet de soins intégrés chez les patients âgés atteints de maladies chroniques est actif au CHRU avec un suivi par télémédecine en soins de santé primaires (en cours). Ce projet est fondé sur une offre de soins innovante et coordonnée pour les maladies chroniques et la gériatrie (clinique CAMAC des comorbidités, clinique des chutes, clinique des maladies neurodégénératives chroniques, clinique de l'appareil locomoteur, clinique du VIH) [15]. La clinique des comorbidités est originale car elle permet d'évaluer l'état d'un patient atteint de maladie chronique en une journée et de proposer une approche thérapeutique en association avec les soins de santé primaire. L'informatisation du système est opérée par le système DXCare $^{\circledR}$ et IPSoins ${ }^{\circledR}$ [16] avec interopérabilité régionale $\left(\right.$ Antares $\left.{ }^{\circledR}\right)$;

DeProPASS (CHRU de Nîmes, médecins généralistes). Un des problèmes de santé de la région est le manque de moyens médicaux dans les cantons de l'arrière-pays. En partenariat avec les maisons médicales du Gard, le CHU de Nîmes propose un dépistage des maladies chroniques à distance (projet DeProPASS). Il est en ligne avec la clinique des comorbidités du CHRU de Montpellier. Une " valise " maladies chroniques est en gestation et un médecin pourrait se déplacer pour " aller vers les patients » des zones peu médicalisées pour détecter les maladies chroniques. Les patients seraient alors suivis par télémédecine. Ce projet pilote permettra d'étendre I'offre de soins aux autres maisons de santé pluri-professionnelles de la région et de couvrir les zones peu médicalisées pour un maillage régional de l'offre de soins ;

- parcours de soins intégrés incluant les urgences (CHRU de Nîmes, Montpellier). L'avenir de la prise en charge optimisée et humanisée des patients ayant une maladie chronique passe par l'organisation du parcours de soins chaque fois que
I'urgence n'est pas vitale en favorisant les filières de soins qui évitent les structures d'urgence, et en prévenant la décompensation sévère par une prise en charge régulière du patient et le recours à l'hospitalisation programmée à délai court [17] ; - fonction érectile et dépistage des maladies chroniques (CHRU de Nîmes, MG). La dysfonction érectile est fréquente dans les maladies métaboliques [18] et cardiovasculaires [19]. Elle est souvent présente lors de la découverte d'un diabète [20] et pourrait représenter un marqueur de maladie chronique et de sa sévérité [19]. Un réseau ville-hôpital est actif [21] et la clinique de comorbidité sera impliquée pour détecter les maladies chroniques chez des patients dépistés en soins de santé primaire ;

- parcours de soins intégrés pour les maladies respiratoires chroniques. Le Centre collaborateur de l'OMS pour l'asthme et la rhinite (CHRU de Montpellier) a initié, il y a environ 10 ans, une prise en charge multisectorielle concernant l'asthme et la rhinite (comorbidités) [22]. Six invitations pour action ont été acceptées par l'Union européenne pour établir un parcours de soins innovant et intégré faisant appel à la télémédecine : LR, Finlande, Norvège, Pologne, Portugal, Turquie ;

- santé bucco-dentaire. Elle est très importante pour les maladies chroniques et le vieillissement [14]. Il existe des barrières pour délivrer des soins appropriés chez les sujets âgés et les populations défavorisées [23]. Un système de téléconsultation (e-Dent) est testé en LR pour améliorer la santé bucco-dentaire des sujets âgés ;

- maladies infectieuses chroniques. Un nombre important de maladies infectieuses sont chroniques (tuberculose, $\mathrm{VIH}$, plaies) [14]. Le site régional est un des centres français les plus actifs dans ces pathologies avec une interaction cliniquerecherche remarquable. La résistance aux antibiotiques est une des priorités nationales;

réseau hépatites LR. La survie des patients atteints d'hépatite C chronique a considérablement augmenté, en particulier en raison de la mise à disposition de thérapeutiques innovantes [24]. Cependant, ces traitements peuvent avoir des effets secondaires [25] et une observance insuffisante est notée, en particulier dans les populations défavorisées [26]. L'efficacité du traitement est potentialisée par l'éducation thérapeutique et une détection précoce. Le réseau multidisciplinaire du LR favorise l'accès aux soins, la détection précoce et le suivi du traitement, en particulier par des actions d'éducation avec maillage territorial (réseau hépatites LR, http:// reseau-hepatites-languedoc-roussillon.org) [27] ;

- prédiction et promotion du AHA au cours de la vie entière (UM1). Le vieillissement actif ne débute pas à 60 ou 65 ans mais se prépare au cours de la vie [28]. Les événements in utero et au cours des premières années sont essentiels pour le vieillissement. Les actions de prévention sont certainement plus efficaces que la prise en charge de sujets ayant déjà un 
handicap. Il faut aussi définir les critères du vieillissement actif et en bonne santé au cours du cycle de la vie et les valider dans des cohortes. C'est le but du projet Horizon 2020 PREPARE AGEING (en cours d'évaluation) ;

- mieux vieillir avec un cancer (CRLC-CHRU de Montpellier, associations de patients). Les patients souffrant d'un cancer reçoivent des traitements de plus en plus efficaces mais qui ne sont pas exempts de complications immédiates ou à distance. Par ailleurs, les effets psychologiques d'un cancer induisent souvent des difficultés de réinsertion familiale, sociale (étude Vicam2) et/ou professionnelle. Les cancers sont souvent associés à des facteurs de risque de maladie chronique ou de second cancer. Des mesures doivent être prises à tout âge mais plus particulièrement chez le sujet âgé (cancérogériatrie) ;

- mieux vieillir avec une maladie génétique rare (l'exemple de la mucoviscidose) (CHRU de Montpellier, associations de patients). Le pronostic de la mucoviscidose passe par un diagnostic précoce et une prise en charge régulière et intensive pour réduire les complications infectieuses et métaboliques. L'espérance de vie a été considérablement allongée mais des efforts sont nécessaires pour permettre aux patients de vieillir mieux [29].

\section{Plans d'action C2 et D4 : vie autonome, environnement adapté aux personnes âgées, appartements thérapeutiques et villes amies}

Les différents projets :

- l'étape, pôle autonomie santé (PAS) de la ville de Lattes est un lieu de référence sur les aides techniques et technologiques avec la mise en place d'un appartement thérapeutique pour la formation du personnel aidant les personnes âgées ou handicapées et la formation professionnelle avec I'INRS (Institut national de recherche et de securité). Le PAS se situe sur le périmètre du projet Écocité, projet phare de l'agglomération de Montpellier, qui peut servir de lieu d'expérimentation grandeur réelle ;

- I'Institut méditerranéen des métiers de longévité (I2ML) est une Fondation partenariale établie en 2011 par l'université de Nîmes avec un partenariat public-privé. Il a contribué à l'émergence de la Silver économie régionale. Un Living Lab a visée domotique est fonctionnel ;

- ÉcoCité et Cité intelligente (Smart C3-1-city). Sur 2500 hectares, répartis sur les communes de Castelnau-le-Lez, Lattes, Montpellier et Pérols, Écocité dessine le nouveau cœur de l'agglomération à l'horizon 2020-2030. Elle instaure un nouvel urbanisme durable, novateur et ambitieux, au sein duquel les transports en commun, les commerces, les logements sociaux, les bureaux, les espaces de partage et de nature sont partie intégrante de la cité. Ses futurs quartiers, imaginés à partir d'une zone urbaine mixte (logements, commerces, activités. . .), desservis par un ensemble de transports en commun performants, équipés d'un réseau de chaleur, comptent un minimum de $25 \%$ de logements sociaux, des équipements majeurs, des espaces naturels et ils présentent la particularité d'assurer la continuité urbaine au piéton comme au cycliste. La commune de Lattes développe un projet santé propre ;

- les thermes de Balaruc-Les-Bains. La société publique locale d'exploitation (SPLE) des thermes de Balaruc-Les-Bains est une société anonyme à capitaux publics (85\% commune de Balaruc, $14 \%$ Thau agglo, $1 \%$ conseil général 34). Elle a pour objet la délégation de service publique DSP avec activité thermale (prise en charge des curistes) et activités complémentaires (bien-être et cosmétique). Les orientations thérapeutiques sont la rhumatologie et phlébologie [30-32]. Le nombre de curistes en 2013 a été de 45650 (8,7 \% de l'activité française). Plus de $75 \%$ des curistes étaient âgés de 60 à 79 ans. Un nouvel établissement ( capacité d'accueil de 4200 curistes/jour) a ouvert en novembre 2014 avec un espace promotion de la santé. Depuis 5 ans, une réunion multidisciplinaire médico-chirurgicale annuelle est organisée, en partenariat avec la Société française de phlébologie ;

- fédération ADMR Gard. À l'échelle nationale, le réseau ADMR est le premier réseau associatif de proximité. L'ADMR est en France la référence du service à la personne depuis près de 70 ans (http://www.admr.org). Elle regroupe dans notre pays 3300 associations sur l'ensemble du territoire avec 110000 bénévoles et 103000 salariés qui agissent chaque jour pour les personnes et avec elles pour apporter un service à domicile sur mesure dans quatre domaines: autonomie, services de confort à domicile, famille et santé. La Fédération ADMR du Gard, pour sa part, consacre 11 millions $€$ par an de son budget à la branche personnes âgées et handicapées. L'implication de I'ADMR dans le projet MACVIA-LR se situe au niveau sociétal (accompagnement des personnes fragiles par des visites régulières), médical (réduction des chutes chez des personnes à risque par l'intervention de l'aide à domicile), et économique (réduction du recours aux soins pour les personnes fragiles et en perte d'autonomie).

\section{Living Labs MACVIA-LR ${ }^{\circledR}$}

\section{Le concept Living Lab}

La recherche doit être appliquée aux soins, aux mesures de prévention et de promotion de la santé, aux actions sociales et au développement économique. Une dissémination de ces thématiques à l'ensemble du territoire doit permettre une promotion du vieillissement actif de toute la population, mais aussi de réduire et mieux appréhender le handicap.

Le concept du Living Lab semble adapté à la problématique. Selon I'European Network of Living Labs (ENOLL, www. openlivinglabs.eu), " un Living Lab regroupe des acteurs publics, privés, des entreprises, des associations, des acteurs individuels, dans l'objectif de tester grandeur nature des 
TABLEAU II

Les Living Labs MACVIA-LR ${ }^{\circledR}$

\begin{tabular}{|c|c|c|c|c|c|}
\hline & & Living Lab & Responsables & Localisation & Organisation \\
\hline \multirow[t]{10}{*}{ Santé et médecine } & 1 & Chute et équilibre & $\begin{array}{l}\text { H. Blain, } \\
\text { A. Dupeyron }\end{array}$ & $\begin{array}{c}\text { Montpellier, Nîmes, } \\
\text { Hérault }\end{array}$ & CHRU de Montpellier, Nîmes \\
\hline & 2 & AVC & D. Hève & Région LR & ARS \\
\hline & 3 & Fragilité & M. Noguès & Région LR & CARSAT \\
\hline & 4 & Maladies chroniques & $\begin{array}{c}\text { A. Avignon, P. Fesler, } \\
\text { D.J. Costa }\end{array}$ & Région LR & CHRU et médecins généralistes \\
\hline & 5 & $\begin{array}{c}\text { Maladies respiratoires } \\
\text { chroniques }\end{array}$ & J. Bousquet, D. Caimi & International & EIP on AHA \\
\hline & 6 & Hépatites & D. Larrey & Région LR & CHRU de Montpellier \\
\hline & 7 & $\begin{array}{c}\text { Maladies infectieuses } \\
\text { chroniques }\end{array}$ & J. Reyne, D. Morquin & Région LR & CHRU de Montpellier \\
\hline & 8 & Handicap & $\begin{array}{l}\text { I. Laffont, } \\
\text { J.Y. Pélissier }\end{array}$ & Région LR & CHRU de Montpellier, Nîmes \\
\hline & 9 & Bien vieillir avec un cancer & M. Ychou & Région LR & CRLC, CHRU de Montpellier \\
\hline & 10 & $\begin{array}{c}\text { Bien vieillir avec une } \\
\text { maladie rare }\end{array}$ & R. Chiron & Montpellier & CHRU, Association mucoviscidose \\
\hline \multirow[t]{3}{*}{ Autonomie et santé } & 11 & L'étape & E. Pastor & Lattes & cCAS Lattes \\
\hline & 12 & $12 \mathrm{ML}$ & C. Laurent & Nîmes & Fondation partenariale \\
\hline & 13 & Thermes de Balaruc & J. Burille & Balaruc-les-bains & $\begin{array}{c}\text { Société publique locale } \\
\text { d'exploitation }\end{array}$ \\
\hline Santé et innovation & 14 & Digi-Health & D. Laune & Montpellier & \\
\hline
\end{tabular}

CAS : centre d'action sociale.

TABLEAU III

Intégration des plans d'action de l'EIP on AHA dans Living Labs « santé et médecine " MACVIA-LR ${ }^{\circledR}$

\begin{tabular}{|c|c|c|c|c|c|c|}
\hline Plan d'action de l'EIP on $A H A$ & A1 & $\mathrm{A} 2$ & A3 & B3 & $\mathrm{C} 2$ & D4 \\
\hline Chutes & ++ & +++ & + & + & ++ & ++ \\
\hline Accident vasculaire cérébral & ++ & ++ & ++ & ++ & + & + \\
\hline Fragilité & + & + & +++ & + & + & + \\
\hline Maladies chroniques & + & + & + & +++ & + & + \\
\hline Urgences et maladies chroniques & + & & + & +++ & & \\
\hline Maladies respiratoires chroniques & + & & ++ & +++ & & + \\
\hline Maladies infectieuses chroniques & ++ & & ++ & +++ & & \\
\hline Hépatites & + & & + & +++ & & \\
\hline Handicap & + & +++ & +++ & +++ & + & +++ \\
\hline Prédiction et promotion du AHA au cours de la vie entière & + & + & + & ++ & + & + \\
\hline Bien vieillir avec un cancer & +++ & + & +++ & +++ & + & ++ \\
\hline Bien vieillir avec une maladie rare & +++ & & +++ & +++ & + & ++ \\
\hline
\end{tabular}




ENCADRÉ 2
Caractéristiques communes des Living Labs « santé et
médecine " MACVIA-LR
Les Living Labs MACVIA-LR ${ }^{\circledR}$ :
- ont été initiés en raison de problèmes de santé publique et/ou
de leur impact dans le vieillissement actif et/ou le handicap
- ont une composante santé qui répond aux attentes des citoyens
pour leur permettre de mieux prendre en charge leur pathologies
et de mieux préparer leur vieillissement actif et en bonne santé
- ont été initiés avec les utilisateurs (patients, citoyens, partenaires
industriels)
- incluent des partenaires publics et privés dont des établissements
publics administratifs (ARS, CARSAT, CPAM)
- ont une composante recherche d'excellence
- dispensent un enseignement innovant permettant de disséminer
les informations à l'ensemble des acteurs concernés incluant les
patients
- fonctionnent en réseau local ou régional avec des interactions
nationales et internationales.
- sont ancrés dans le tissu associatif (patients)
- doivent permettre un maillage territorial pour l'ensemble des
citoyens
- ont une évaluation médico-économique de leur impact
- sont réévalués et optimisés régulièrement
ARS : Agence régionale de santé ; CARSAT : caisse d'assurance
retraite et de la santé au travail ; CPAM : caisse primaire
d'assurance maladie.

services, des outils ou des usages nouveaux. Il s'agit de sortir la recherche des laboratoires et les soins des centres hospitaliers pour les faire descendre dans la vie de tous les jours, en ayant souvent une vue stratégique sur les usages potentiels des nouvelles approches et technologies. Une coopération entre des collectivités locales, les acteurs de santé et de la vie sociale, les entreprises et des laboratoires de recherche doit être mise en place avec les utilisateurs potentiels. II s'agit de favoriser l'innovation ouverte, partager les réseaux et impliquer les utilisateurs dès le début de la conception ".

\section{Les Living Labs MACVIA-LR ${ }^{\circledR}$}

Le site de référence $M A C V I A-L R^{\circledR}$ répond à l'ensemble du plan d'action de l'EIP on AHA par des actions verticales qui ont été regroupées en activités transversales, complémentaires et synergiques sous la forme de plusieurs Living Labs et constituent l'institut EURICAP. Trois types de Living Labs ont été initiés (tobleau II).

Les Living Labs « santé et médecine » MACVIA-LR ${ }^{\circledR} 1,2,4,5$ et 6 correspondent aux critères ENOLL et aux plans d'action de l'EIP on AHA (tableau III). Tous les Living Labs MACVIA-LR ${ }^{\circledR}$ ont des caractéristiques communes (encadré 2 et figure 3).

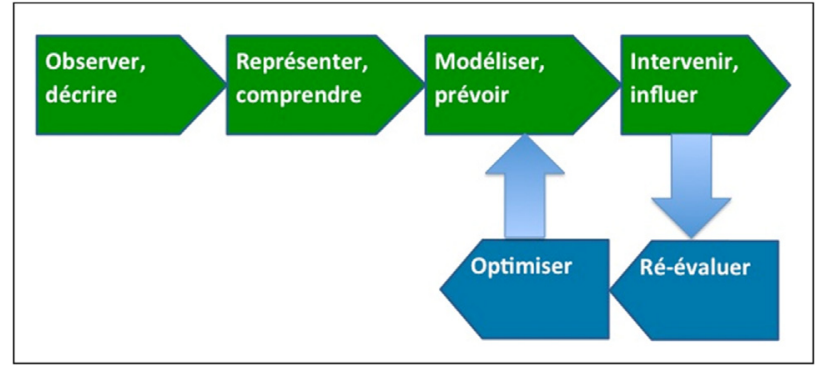

FIGURE 3

Organisation commune des Living Labs MACVIA-LR ${ }^{\circledR}$ (d'après $R$ Picard et P Gleyze, modifié)

\section{EURICAP (EURopean Institute for Chronic diseases, Ageing and handicaP)}

\section{objectifs}

L'institut EURICAP regroupe les Living Labs MACVIA-LR ${ }^{\circledR}$, site de référence de l'EIP on $A H A$, et propose un projet innovant, en réseau avec maillage territorial pour (figures 4 et 5 ) :

- partager les connaissances à tous les niveaux sur un problème de santé publique reconnu ;

- promouvoir le vieillissement actif et prévenir le handicap pour réduire les inégalités sociales ;

- impliquer les utilisateurs de l'ensemble de la région LR ;

- renforcer les synergies entre les différents Living Labs et réduire la fragmentation ;

- renforcer le site de référence MACVIA-LR ${ }^{\circledR}$;

- permettre le développement économique ;

- créer une synergie pour les PME du domaine de la santé en LR ;

- ouvrir les Living Labs et EURICAP à des partenaires nationaux et internationaux.

L'institut EURICAP est fondé sur 4 thèmes principaux (encadré 3).

\section{Recherche, pôle d'excellence d'EURICAP}

La recherche d'EURICAP est innovante car elle combine une recherche transversale d'excellence avec le patient au centre de la réflexion, pour mieux comprendre les maladies chroniques et le vieillissement, prendre en charge les patients et proposer des stratégies de prévention innovantes ainsi que de nouvelles cibles thérapeutiques (figure 6).

\section{Sujets phénotypés}

La recherche est d'abord fondée sur une offre de soins avec maillage territorial et l'inclusion de patients parfaitement phénotypés, suivis par télémédecine dans des cohortes de la Région LR ou dans celles dont les membres de MACVIA-LR ${ }^{\circledR}$ sont des participants actifs (MeDALL, 3 cités...). Une optimisation des soins intensifs est indispensable pour atteindre les objectifs. 


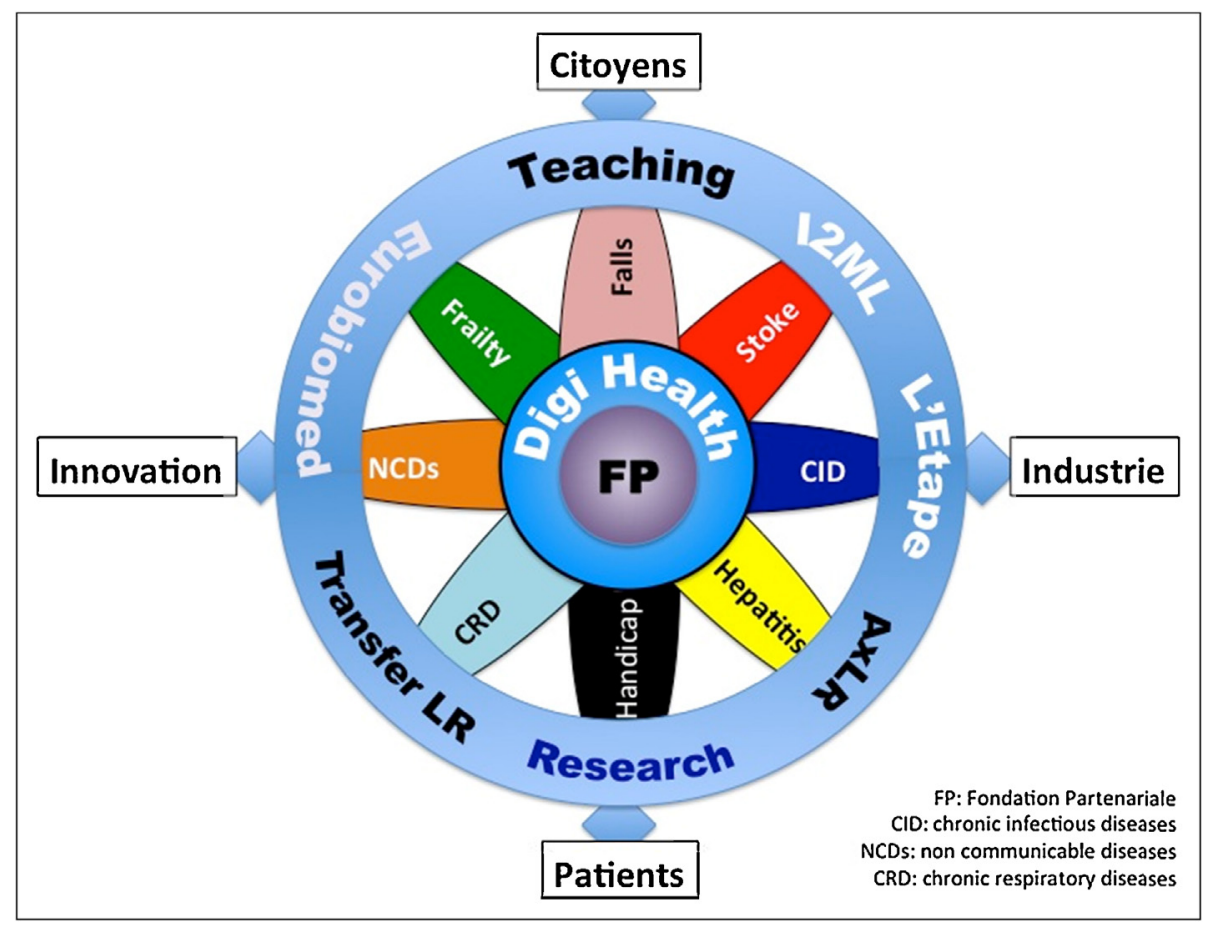

FIGURE 4

Institut EURICAP

\section{Centre d'investigation clinique (CIC 1411), CHRU de Montpellier}

Plateforme d'investigation clinique ouverte aux cliniciens, aux chercheurs fondamentalistes et aux promoteurs industriels et institutionnels, le CIC constitue un lieu d'expertise et de savoirfaire. Les moyens humains et techniques mis à disposition permettent de mener une recherche clinique conforme aux exigences de l'assurance qualité, dans le respect des règles éthiques et juridiques, aboutissant à une valorisation optimale.

\section{Plateformes mutualisées}

La recherche fait ensuite appel à des plateformes biologiques, omiques et d'imagerie déjà en place sur le site et mutualisées. Le projet BioBanques $L R$ a été créé pour mutualiser les moyens et les compétences des principaux établissements de santé régionaux. BioBanques LR propose de mettre en place un portail unique des collections des centres collecteurs des échantillons biologiques humains des CHRU de Montpellier et de Nîmes et de I'ICM-Val d'Aurelle) dans les conditions de qualité requises et le respect de la réglementation.

\section{Axes de recherche prioritaires}

Ils sont représentés par les points forts du site MontpellierNîmes regroupés autour des maladies inflammatoires et chroniques, des neurosciences et des maladies infectieuses chroniques et du mouvement (EUROMOV).

\section{Aspects innovants}

Un axe thérapeutique innovant couplé au projet est l'Institut de biothérapie pour la médecine régénératrice. Les associations de patients sont associées à la recherche pour mieux comprendre leurs besoins et mieux les intégrer à la recherche. L'ensemble des données (dès l'inclusion des sujets) sont intégrées par TIC et une analyse bio-informatique est disponible.

Les structures de valorisation de la région LR sont intégrées aux projets de recherche.

\section{La Structure fédérative de recherche administration, sociologie et médico-économie (SFR ASMES) du CHRU de Montpellier (R. Bourret, G. Delande)} Cette structure :

- propose d'intégrer les problèmes éthiques, juridiques, économiques et institutionnels avec les nécessités de la recherche clinique, translationnelle et fondamentale, en particulier les bases de données et les données omics :

- pour un environnement scientifique coordonné et transversal afin d'assurer une politique de soutien et de renforcement de la recherche pour le CHRU de Montpellier, au plus près du " terrain », dans une logique de mixité et de diversité ; 


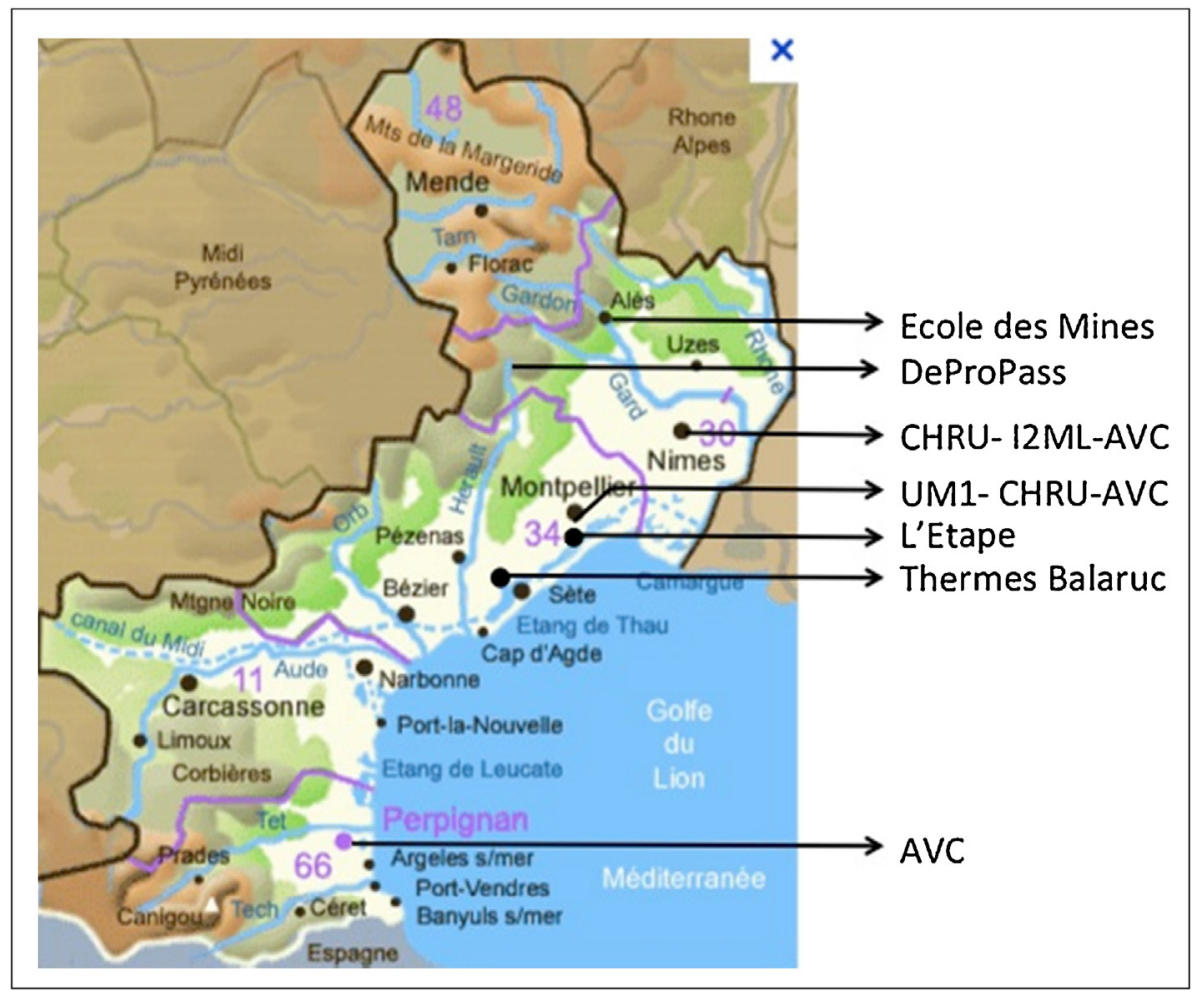

FIGURE 5

Sites des Living Labs MACVIA-LR ${ }^{\circledR}$

- est une valorisation plus rapide de la recherche en lien étroit avec les organismes de valorisation de la région.

La SFR est localisée à l'hôpital La Colombière. Elle réunit les équipes dans les domaines de la santé en droit, de la sociologie, des sciences humaines et sociales, des sciences de gestion et

\section{ENCADRÉ 3}

Thèmes principaux de l'institut EURICAP

1. Préparer le vieillissement actif

- Définir et modéliser le vieillissement actif

- Bien vieillir avec un cancer

- Bien vieillir avec une maladie rare

- Prévenir la fragilité

2. Maladies chroniques

- Maladies chroniques avec comorbidité

- Maladies respiratoires chroniques

- Maladies infectieuses chroniques et hépatites

- Santé bucco-dentaire

3. Chutes et équilibre

- Chutes

- AVC

4. Handicap administrative, des TICS, du médico-économie, du paramédical et du médical en relation directe (par projet) avec les chercheurs et les cliniciens.

\section{Les projets de recherche}

Un recensement des projets de recherche a été effectué sur les sites de Montpellier et de Nîmes (UM1, UM2, Inserm, CNRS, CHRU de Montpellier et Nîmes). Plus de 80 projets portés par des structures de recherche ont été décrits. Les financements acquis sont d'environ 45 millions $€$ et les financements demandés environ 19 millions $€$ (tableau IV). La liste complète des projets est présentée dans le site web MACVIA-LR ${ }^{\circledR}$ (http:// macvia.crlanguedocroussillon.fr).

\section{EDI : EURICAP Data Integration}

L'ensemble des données recueillies dans les différents projets ne peuvent être valorisées qu'à travers une structure forte de data-management et de data intégration. C'est l'objectif de la mise en place du plateau informatique chez Digi-Health, dédié à la réconciliation de données harmonisées contextualisées et rendues cohérentes et comparables. Ainsi, suivis cliniques, observations environnementales et données à haute dimension (données omics) pourront être accessibles et compréhensibles à partir d'une seule plateforme. Cette structure accentuera la transversalité des projets Living Labs. 


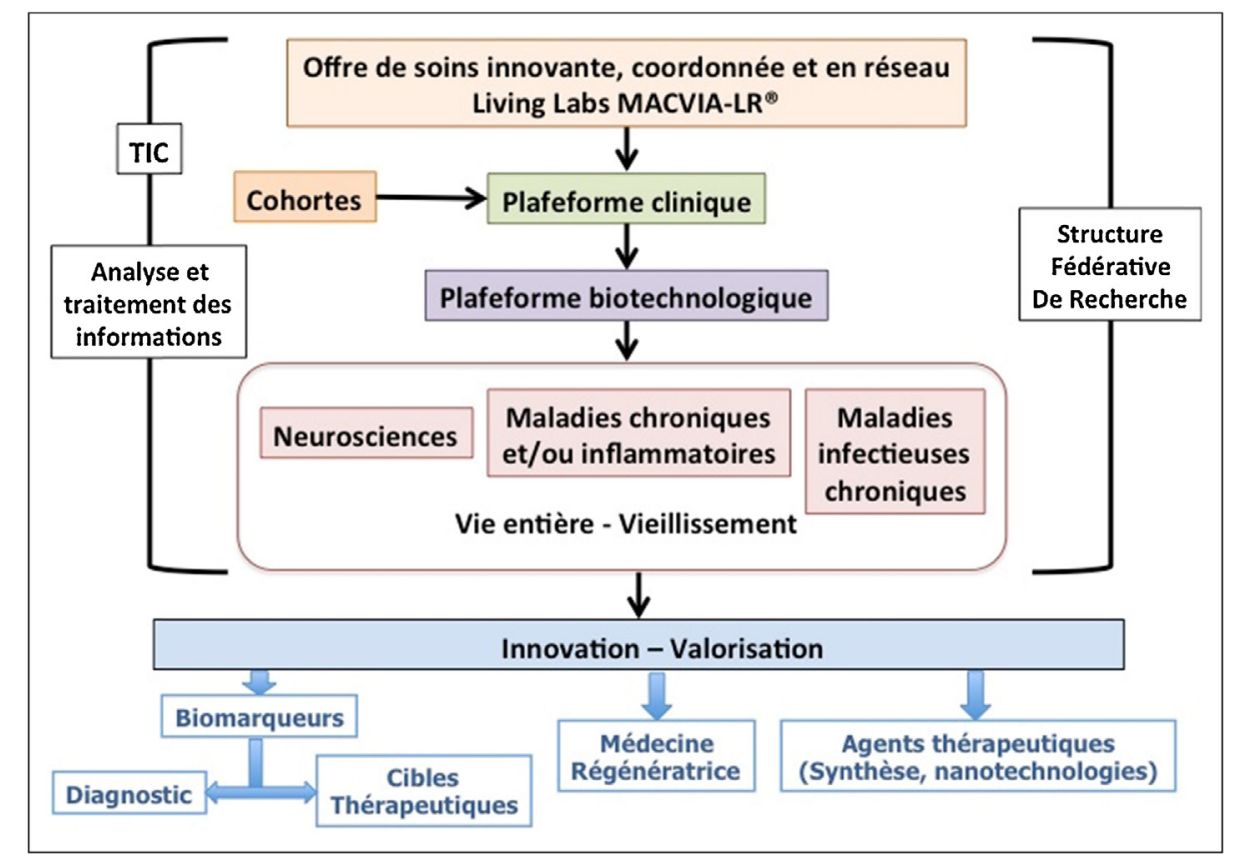

FIGURE 6

Interactions entre les différentes composantes de MACVIA-LR ${ }^{\circledR}$ et la recherche

\section{Enseignement et dissémination : les atouts d'EURICAP}

L'enseignement est un atout essentiel du site de référence (tableau V). Il s'adresse à tous les partenaires du projet et en particulier aux patients. Les associations de patients sont impliquées dans ce projet pour une meilleure connaissance des maladies chroniques sur le plan de la prévention et de la prise en charge, et, pour un engagement des patients dans l'innovation. Ce projet sera fondé sur les enseignements d'excellence de

TABLEAU IV

Activités de recherche liées à EURICAP

\begin{tabular}{lcc} 
Living Labs EURICAP & $\begin{array}{c}\text { Recherche } \\
\text { directement } \\
\text { associée } \\
\text { au projet }\end{array}$ & $\begin{array}{c}\text { Recherche } \\
\text { moyennement } \\
\text { ou faiblement } \\
\text { associée au } \\
\text { projet }\end{array}$ \\
\hline Chutes & 8 & 23 \\
\hline Maladies chroniques & 32 & 41 \\
\hline Accident vasculaire cérébral & 7 & 1 \\
\hline Maladies infectieuses chroniques & 12 & 3 \\
\hline Handicap & 13 & 45 \\
\hline Fragilité, vieillissement & 24 & 42 \\
\hline
\end{tabular}

la région et sur les programmes innovants de télémédecine. II représentera un programme transversal pour les professionnels de santé (enseignement universitaire et post-universitaire), les chercheurs et les travailleurs sociaux incluant en particulier le projet Trans-Innov-Longévité (ANR).

Auprès du grand public et des médias, les termes " maladie chronique » et "vieillissement actif et en bonne santé » sont mal connus. Il est donc indispensable d'offrir un programme transversal pour mieux faire comprendre ces maladies, leurs comorbidités et facteurs de risque. II convient aussi d'intégrer dans ce projet la notion de "vieillissement actif et en bonne santé » qui est associée aux maladies chroniques. L'activité physique et la nutrition seront promues.

\section{Autonomie santé au service des patients}

ÉTAPE, I2ML, Balaruc : EURICAP dispose dans ses Living Labs de laboratoires dédiés aux patients handicapés ou fragiles utilisant souvent les nouvelles technologies pour adapter des aides techniques (aides au déplacement, à la communication, vision, audition) et favoriser leur autonomie.

Fédération ADMR du Gard : elle consacre, pour sa part, 11 millions € par an de son budget à la branche personnes âgées et handicapées.

\section{EURICAP, un institut européen}

Les activités d'EURICAP en LR seront complétées par des actions multilatérales en France et en Europe, par exemple, HARC 


\begin{tabular}{|c|c|c|}
\hline Training of health care professionals & UM1 & $\begin{array}{l}\text { Pre and post-graduate training of the oldest medical school in } \\
\text { the Western world with specific courses of AHA (primary care, } \\
\text { specialists, geriatrics) }\end{array}$ \\
\hline $\begin{array}{l}\text { Kinésithérapie - ergonomie } \\
\text { Orthophonie (C. Hérisson) }\end{array}$ & UM1 & Training of professionnals with a special course for AHA \\
\hline Euromov & - & - \\
\hline $\begin{array}{l}\text { Institut transdisciplinaire } \\
\text { d'étude du vieillissement } \\
\text { (J.-M. Verdier) }\end{array}$ & $\begin{array}{l}\text { École pratique des } \\
\text { Hautes Études }\end{array}$ & $\begin{array}{l}\text { Two teaching courses are operative in collaboration with the } \\
\text { CNAM : (i) professional care givers of elderly people and (ii) } \\
\text { nurses in nursing homes }\end{array}$ \\
\hline $\begin{array}{l}\text { L'étape } \\
\text { (E. Pastor) }\end{array}$ & $\begin{array}{l}\text { INRS teaching (Institut national } \\
\text { de recherche et sécurité) }\end{array}$ & $\begin{array}{c}\text { One teaching unit consists of an educational apartment and } \\
\text { courses. Training, in particular for the prevention of disability } \\
\text { at work }\end{array}$ \\
\hline $\begin{array}{l}\text { Master, ICT and health } \\
\text { (G. Dray, M. Hayot) }\end{array}$ & $\begin{array}{l}\text { UM1, UM2, école des mines d'Alès, } \\
\text { institut Télécom }\end{array}$ & $\begin{array}{c}\text { Students from ICT, physics, biology, medicine, pharmacy or } \\
\text { sports and citizens }\end{array}$ \\
\hline $\begin{array}{l}\text { Trans-Innov-Longévité } \\
\text { (C. Jeandel) }\end{array}$ & $\begin{array}{l}\text { Investissement d'avenir } \\
\text { ANR } \\
\text { UM1 }\end{array}$ & $\begin{array}{l}\text { Trans-disciplinary, multisectoral, private-public partnership to } \\
\text { train and coach on frailty, ageing and independent living. ICT } \\
\text { teaching in } 3 \text { universities (Paris, Montpellier, Strasbourg: } \\
\text { http://www.aunege.fr) and French speaking countries. Master } \\
\text { of gerontology. }\end{array}$ \\
\hline Digi-Health (D. Laune) & $\begin{array}{c}\text { PIA } \\
\text { Filières industrielles }\end{array}$ & Training center for patients, caregivers and health professionals \\
\hline \multicolumn{3}{|l|}{ Coaching of patients } \\
\hline Balaruc-les-Bains (J. Burille) & Société française phlébologie & Ateliers annuels \\
\hline
\end{tabular}

ANR : Agence nationale de la recherche ; UM1 : université Montpellier 1 ; UM2 : université Montpellier 2 ; ICT : technologies de l'information et des communications.

(Healthy Ageing Research Center, Lodz, Pologne, http://harc. umed.pl) dirigé par M Kowalski.

Ainsi, EURICAP peut être le centre français d'activités complémentaires dans d'autres sites que MACVIA LR avec une gouvernance tenant compte de ces interactions.

\section{Des indicateurs précis et mesurables Healthy Life Years}

L'EIP on AHA propose des indicateurs pour mesurer l'efficacité du programme, en particulier le HLY (Healthy Life Years) [3]. Cependant, il est difficile d'obtenir des valeurs au niveau régional. L'utilisation de cet indicateur en région LR est en discussion, en collaboration avec la Joint Action EHLEIS (European Health \& Life Exeptancy information system: www.healthindicators.eu).

\section{Hospitalisations évitables}

L'échec de la prévention et l'accès inadapté aux soins de santé primaires se traduisent par de nombreuses hospitalisations coûteuses qui pourraient être évitées. Les hospitalisations évitables représentent un indicateur précis de l'efficience du système de santé qui peut être mesuré au niveau local, régional ou national [33-37]. Un des objectifs de l'EIP on AHA est la diminution des hospitalisations non programmées pour maladies chroniques de $30 \%$ en 2020. Ce pourcentage de réduction est ambitieux mais raisonnable et facilement mesurable. L'étude de cet indicateur est en cours dans MACVIA-LR ${ }^{\circledR}$ pour établir une évaluation des programmes mis en place, en particulier pour les maladies chroniques et les chutes.

\section{Fragilité}

Un atlas de la fragilité en Languedoc-Roussillon chez les retraités de 55 à 74 ans du régime général a été établi en 2012. Il détaille I'analyse spatiale et l'évolution temporelle de la fragilité [38]. II servira d'un part de données de base à MACVIA-LR ${ }^{\circledR}$ mais aussi à déterminer les zones géographiques où il faudra avoir le plus d'impact.

\section{Innovation et interactions d'EURICAP avec le tissu industriel \\ Les structures régionales institutionnelles}

La région LR est le site de référence de l'EIP on AHA et apporte un soutien très fort au projet MACVIA-LR ${ }^{\circledR}$ et EURICAP. 
L'UM1 est porteur de la Fondation partenariale MACVIA avec la région LR et les CHRU de Montpellier et Nîmes. Cette fondation représente la structure juridique à laquelle MACVIA-LR ${ }^{\circledR}$ et EURICAP sont adossés.

L'association Transferts LR (http://www.transferts-Ir.org) est financée par l'Europe, l'État (cellule de diffusion technologique) et la région LR. Elle bénéficie du soutien des conseils généraux du Gard, de l'Hérault, de la Lozère, des Pyrénées orientales et des communautés d'agglomérations de Montpellier et Nîmes. En 7 ans, avec l'aide des financeurs, Transferts LR a :

- conseillé 2000 entrepreneurs ;

- accompagné 1000 projets de R\&D ;

- mis en relation 1200 PME régionales avec des centres de compétences technologiques ;

- évalué pour des porteurs de projet, 260 études de marché ;

- émis 650 avis COSTI (conseils d'orientation scientifiques, techniques et industriels) ;

- formé 290 conseillers dans le cadre du Réseau régional d'innovation.

Le Réseau régional spécialisé dans l'accompagnement et la création des entreprises innovantes, SYNERSUD (http://www. synersud.com), fédère 21 structures d'accompagnement à la création et au développement d'entreprises innovantes en Languedoc-Roussillon. Depuis sa création, SYNERSUD a contribué à la création de 2200 start-ups qui ont généré plus de 8000 emplois. Les taux de survie de ces entreprises sont remarquables : $91 \%$ à 3 ans et $85 \%$ à 5 ans. Le réseau SYNERSUD est composé de Pépinières d'entreprises, de Maisons des entreprises, de CEEI (Centres européens d'entreprises et d'innovation) ou BIC (Business Innovation Center), de technopoles et d'incubateurs technologiques de la région LR. L'association SYNERSUD bénéficie du soutien financier de la région $L R$.

\section{SATT AXLR}

AxLR (www.axlr.com) est la société d'accélération du transfert de technologie (SATT) du LR. Elle a pour ambition de valoriser la recherche publique et le transfert de technologie vers l'industrie. AxLR a été créée le $1^{\mathrm{er}}$ août 2012 à la suite de l'appel à projets du programme investissements d'avenir. Sa capacité d'investissement s'élève à 50 millions d'euros et sera progressivement augmentée.

AxLR souhaite améliorer significativement les résultats de la recherche publique, que ce soit sous forme de licences, de partenariats industriels, de création d'entreprises ou en facilitant la mobilité des chercheurs. Ses principaux objectifs sont les suivants :

- financer la maturation des projets issus des laboratoires de recherche vers les entreprises;

- financer le dépôt des brevets et autres droits ;

- développer le transfert de l'innovation et des savoir-faire vers le monde économique pour favoriser la création de valeur.

\section{BIC (Business Innovation Centre) de Montpellier}

Le Business \& Innovation Centre de Montpellier agglomération est en parfaite synergie avec la recherche, l'entreprise et les initiatives du secteur public pour accompagner la création et le développement d'entreprises innovantes. Il est membre du réseau Synersud. Créé en 1987, agréé Business \& Innovation Center en 1988 par l'Europe, il a reçu l'Award International 2007 du «Meilleur Incubateur " décerné par NBIA. Depuis 1987, 542 entreprises ont été créées (4400 emplois en 2014) avec un taux de pérennité de plus de $80 \%$ et un solide réseau de partenaires : universités, centres de recherche, Oséo, Languedoc-Roussillon Incubation, investisseurs publics et privés, collectivités, consultants.

\section{Eurobiomed}

Eurobiomed (www.eurobiomed.org), le pôle de compétitivité de la filière santé regroupant les régions Provence Alpes Côte d'Azur et LR, a déposé un Commitment for Action dans le cadre de MACVIA-LR ${ }^{\circledR}$ auprès de l'EIP on AHA. Eurobiomed regroupe le pôle de compétitivité ORPHEME et les clusters Holobiosud et Bioméditerranée en s'appuyant sur les synergies préexistantes entre ces trois associations au service du développement des activités innovantes dans le domaine de la santé et des Sciences du vivant.

Avec un réseau de plus de 400 entreprises, majoritairement des PME innovantes, 8 universités et de nombreuses écoles formant 39000 étudiants, 400 laboratoires de recherche, 4 CHRU et une Direction interrégionale des recherches cliniques, le cluster Eurobiomed se positionne au rang des principaux clusters français et européens en santé en termes de nombre d'entreprises, de recherche et d'excellence scientifique et d'équipements dédiés à l'innovation et à la création d'entreprises innovantes. Dans le cadre de la labellisation de projets de recherche et développement, Eurobiomed accompagne le montage de projets R\&D. Eurobiomed aide ainsi les porteurs de projets à soumettre leurs projets afin d'obtenir la labellisation par le pôle de compétitivité. Ses objectifs sont :

- de fédérer et développer les synergies entre les entreprises, laboratoires publics, structures de formation et d'accompagnement des régions PACA et $L R$, par la mise en place d'actions d'animation de réseau et le développement de projets partenariaux ;

- de développer de nouvelles innovations au service de la santé ;

- de renforcer la visibilité du réseau et des entreprises ;

- d'accompagner le développement des entreprises et de l'emploi.

\section{Digi-Health (ex CR2i)}

Porté par le pôle de compétitivité Eurobiomed, le dossier régional d'un centre de recherche et d'innovation industrielle (CR2I, renommé Digi-Health), dédié au secteur TIC et santé, et basé 
à Montpellier, figure parmi les 4 projets retenus le 27 février 2012 dans le cadre du programme investissements d'avenir (Grand Emprunt), sur le volet des «platesformes mutualisées d'innovation » (PFMI). Ce projet a été transféré en 2013 sur le programme "filières industrielles » lancé par le ministère de I'Industrie et Bpifrance et destiné à renforcer la compétitivité et l'efficacité des filières industrielles stratégiques françaises en soutenant des actions structurantes matérielles ou immatérielles. Il vise à rapprocher diagnostic médical et nouvelles technologies. Le programme se concrétisera sous la forme d'un centre d'expertise rassemblant sur le même site des acteurs du diagnostic, de la thérapie et des nouvelles technologies. L'objectif de Digi-Health est de développer et d'industrialiser de nouvelles solutions de santé en matière de diagnostic et de télémédecine, notamment dans les domaines de la médecine personnalisée, ambulatoire, et à distance.

Parmi les partenaires associés au projet figurent des grands groupes (IBM, Sanofi, Horiba Medical), des PME (Gosis, Groupe MIT, Amylgen, ASA, Histalim, Horiba Pharma, Skuldtech), I'association Biomeridies, ainsi que des établissements de recherche et d'enseignement supérieur tels que l'UM1, l'école des mines d'Alès, le CHRU de Montpellier, et l'Établissement français du sang.

\section{Interactions avec I'Union européenne Vieillissement actif et en bonne santé au cours du cycle de la vie}

La présidence polonaise du Conseil de l'Europe (2011) a proposé une priorité sur la prise en charge des maladies respiratoires chroniques chez l'enfant pour promouvoir le vieillissement actif et en bonne santé [39]. Les éléments déterminants du développement des maladies chroniques ont été soulignés lors de la Présidence chypriote du Conseil de l'Europe (2012) [40]. Suivant ses recommandations, les membres de MACVIA-LR ont planifié en septembre 2012 trois réunions successives financées par le Conseil régional $L R$ :
- Bruxelles, novembre 2012 (Early determinants of chronic respiratory diseases) : réunion organisée au Parlement européen en collaboration avec la Présidence chypriote [40] ;

- Montpellier, décembre 2013 (Developmental determinants in non-communicable chronic diseases and ageing, from research to novel policies and value creation) (http:// macvia.cr-languedocroussillon.fr). Cette réunion a été co-organisée par le NIH suivant un workshop à Bethesda en septembre 2012 [41] ;

- Montpellier, 20, 21 octobre 2014 : Operative definition of AHA en collaboration avec le Network of Reference Sites of the EIP on $A H A$ and EUGMS (European Union Geriatric Medicine Society).

\section{Réseau européen des sites de référence de l'EIP on AHA}

Trente-deux sites de référence ont été labellisés par l'EIP on AHA. MACVIA-LR ${ }^{\circledR}$ est l'un d'entre eux et représente un des sites les plus actifs du Réseau des sites de références dirigé par I'Irlande du Nord. MACVIA-LR ${ }^{\circledR}$ est le site qui dirige deux projets : AIRWAYS Integrated Care Pathways et la définition opérationnelle de AHA.

\section{Fonds structurels européens}

EURICAP est éligible aux fonds structurels européens, en particulier FEDER 2014-2020, de la région LR. La région LR a défini sa "Smart Specialisation Strategy " (3S), fondée sur 7 domaines d'innovation comme pilier du plan économique de développement voté en décembre 2013. Une des priorités est le diagnostic, les thérapeutiques innovantes et les médecines alternatives appliqués aux maladies chroniques et au vieillissement.

\footnotetext{
Déclaration d'intérêts : Jean Bousquet a reçu des honoraires de :

- Scientific and advisory boards - Almirall, Meda, Merck, MSD, Novartis, Sanofi-Aventis, Takeda, Teva, Uriach;

- Lectures during meetings-Almirall, AstraZeneca, Chiesi, GSK, Meda, Menarini, Merck, MSD, Novartis, Sanofi-Aventis, Takeda, Teva, Uriach; - Board of Directors-Stallergènes.
}

\section{Références}

[1] Rechel B, Grundy E, Robine JM, et al. Ageing in the European Union. Lancet 2013;381: 1312-22.

[2] Morley JE, Vellas B, van Kan GA, et al. Frailty consensus: a call to action. JAMA 2013;14: 392-7.

[3] Jagger C, Gillies C, Moscone F, et al. Inequalities in healthy life years in the 25 countries of the European Union in 2005: a cross-national meta-regression analysis. Lancet 2008;372: 2124-31.

[4] Bousquet J, Anto JM, Sterk PJ, et al. Systems medicine and integrated care to combat chronic noncommunicable diseases. Genome Med 2011;3:43

[5] Bousquet J. [Non-communicable chronic diseases, a global public health priority recognized by the United Nations]. Presse Med 2011;40:787-9.

[6] Barnett K, Mercer SW, Norbury M, Watt G, Wyke S, Guthrie B. Epidemiology of multimorbidity and implications for health care, research, and medical education: a cross-sectional study. Lancet 2012;380:37-43.

[7] Bousquet J, Hajjam J, Piette F, et al. [The French reference sites of the European innovation partnership on active and healthy ageing]. Presse Med 2013;42:1558-61.

[8] Bousquet J, Bourquin C, Augé $P$, et al. MACVIA-LR reference site of the European innovation partnership on active and healthy ageing. Eur Geriatr Med 2014;5: 406-15.

[9] Robine JM, Cambois E, Nusselder W, Jeune $B$, Oyen $H V$, Jagger $C$. The joint action on healthy life years (JA: EHLEIS). Arch Public Health 2013;71:2.

[10] Croswell J, Shin YR. Prevention of falls in community-dwelling older adults: 
recommendation statement. Am Fam Physician 2012;86:1135-6.

[11] Blain H, Abecassis F, Adnet P, et al. Living Lab Falls-MACVIA-LR: the falls prevention initiative of the European innovation partnership on active and healthy ageing (EIP on AHA) in Languedoc Roussillon. Eur Geriatr Med 2014:5:416-25.

[12] Clegg A, Young J, Iliffe S, Rikkert MO, Rockwood K. Frailty in elderly people. Lancet 2013;381:752-62

[13] WHO. Action plan for the global strategy for the prevention and control of non communicable diseases. Prevent and control cardiovascular diseases, cancers, chronic respiratory diseases, diabetes; 2013 [Accès au site le 14/ 02/2015] http://www.who.int/nmh/ publications/ncd action plan en.pdf.

[14] European Union Health Policy Forum. Answer to DG SANCO consultation on chronic diseases: 2012 [Accès au site le 14/02/2015] http:// eceuropaeu/health/interest groups/docs/ euhpf_answer_consultation_jan2012_enpdf; 2012.

[15] Bousquet J, Jorgensen C, Dauzat M, et al. System medicine approaches for the definition of complex phenotypes in chronic diseases and ageing. From concept to implementation and policies. Curr Pharm Des 2014;20:5928-44.

[16] Bourret R, Bousquet J. An integrated approach to telemonitoring noncommunicable diseases: best practice from the European innovation partnership on active and healthy ageing. World Hosp Health Serv 2013;49:25-8.

[17] Claret P, Bobbia B, Richard P, Poher F, de-laCoussaye J. [Surcharge du service des urgences: causes, conséquences et ébauches de solution]. Ann Fr Med Urgence 2014;4:961015.

[18] Redrow GP, Thompson CM, Wang R. Treatment strategies for diabetic patients suffering from erectile dysfunction: an update. Expert Opin Pharmacother 2014;15:1827-36.

[19] Jackson G, Boon N, Eardley I, et al. Erectile dysfunction and coronary artery disease prediction: evidence-based guidance and consensus. Int J Clin Pract 2010;64:848-57.
[20] Corona G, Giorda CB, Cucinotta D, Guida P, Nada E, group S-Ds. The SUBITO-DE study: sexual dysfunction in newly diagnosed type 2 diabetes male patients. J Endocrinol Invest 2013;36:864-8

[21] Costa P, Grandmottet G, Mai HD, Droupy S. Impact of a first treatment with phosphodiesterase inhibitors on men and partners' quality of sexual life: results of a prospective study in primary care. J Sex Med 2013;10:1850-60.

[22] Bousquet J, Addis A, Agache I, et al. Integrated care pathways for airway diseases (AIRWAYS ICPS). EUr Respir I 2014:44:304-23.

[23] Carson SJ, Edwards M. Barriers to providing dental care for older people. Evid Based Dent 2014;15:14-5.

[24] Hezode C, Fontaine H, Dorival C, et al. Triple therapy in treatment-experienced patients with $\mathrm{HCV}$-cirrhosis in a multicentre cohort of the French Early Access Programme (ANRS CO20-CUPIC). J Hepatol 2013;59:434-41 [NCT01514890].

[25] Bota S, Sporea I, Sirli R, et al. Severe adverse events during antiviral therapy in hepatitis C virus cirrhotic patients: a systematic review. World J Hepatol 2013;5:120-6.

[26] Lieveld FI, van Vlerken LG, Siersema PD, van Erpecum KJ. Patient adherence to antiviral treatment for chronic hepatitis B and C: a systematic review. Ann Hepatol 2013;12:380-91.

[27] Larrey D, Salse A, Ribard D, et al. Education by a nurse increases response of patients with chronic hepatitis $\mathrm{C}$ to therapy with peginterferon-alphaza and ribavirin. Clin Gastroenterol Hepatol 2011;9:781-5.

[28] Kuh D, Cooper R, Hardy R, Richards M, BenShlomo Y. A life course approach to healthy ageing. Oxford: Oxford University Press; 2014

[29] Simmonds NJ. Ageing in cystic fibrosis and long-term survival. Paediatr Respir Rev 2013;14(Suppl. 1):6-9.

[30] Mooventhan A, Nivethitha L. Scientific evidence-based effects of hydrotherapy on various systems of the body. North Am J Med Sci 2014;6:199-209.

[31] Forestier R, Desfour H, Tessier JM, et al. Spa therapy in the treatment of knee osteoarthritis: a large randomised multicentre trial. Ann Rheum Dis 2010;69:660-5.
[32] Forestier R, Genty C, Waller B, et al. Crenobalneotherapy (spa therapy) in patients with knee and generalized osteoarthritis: a post-hoc subgroup analysis of a large multicentre randomized trial. Ann Phys Rehab Med 2014;57:213-27.

[33] Bornemeier WC. Avoidable hospitalization. III Med J 1953;104:325-6.

[34] Billings J, Zeitel L, Lukomnik J, Carey TS, Blank AE, Newman L. Impact of socioeconomic status on hospital use in New York City. Health Aff 1993;12:162-73.

[35] Bindman AB, Grumbach $K$, Osmond $D$, et al. Preventable hospitalizations and access to health care. JAMA 1995;274:305-11.

[36] Weissman JS, Gatsonis C, Epstein AM. Rates of avoidable hospitalization by insurance status in Massachusetts and Maryland. JAMA 1992;268:2388-94

[37] Rosano A, Loha CA, Falvo $R$, et al. The relationship between avoidable hospitalization and accessibility to primary care: a systematic review. Eur J Public Health 2013;23:356-60

[38] Direction adjointe assurance maladie et action sociale. Service santé prévention prospective: Carsat (Caisse d'assurance retraite \& santé au travail), assurance retraite assurance maladie. Atlas de la fragilité du Languedoc-Roussillon retraités de 55 à 74 ans du régime général. Analyse spatiale et évolution temporelle. Diagnostic régional, diagnostics départementaux. Observatoire des situations de fragilité des personnes âgées; 2012 [Accès au site le 14/02/2015].

[39] Samolinski B, Fronczak A, Wlodarczyk A, Bousquet J. Council of the European Union conclusions on chronic respiratory diseases in children. Lancet 2012;379:e45-6.

[40] Bousquet J, Tanasescu CC, Camuzat T, et al. Impact of early diagnosis and control of chronic respiratory diseases on active and healthy ageing. A debate at the European Union parliament. Allergy 2013;68: 555-61.

[41] Bousquet J, Gern JE, Martinez FD, et al. Birth cohorts in asthma and allergic diseases: report of a NIAID/NHLBI/MeDALL joint workshop. Allergy Clin Immunol 2014;133:1535-46. 